\title{
UV VARIABILITY OF A LARGE SAMPLE OF AGN
}

\author{
S.PALTANI \\ Institut d'Astronomie de l'Université de Lausanne, CH-1290 Chavannes-des-Bois \\ and \\ T. J.-L. COURVOISIER \\ Observatoire de Genève, CH-1290 Sauverny
}

The IUE-ULDA database (Version 3.0, complete until the end of 1988) contains about 3500 spectra of more than 500 AGN of different classes (see Courvoisier and Paltani, IUE-ULDA Access Guide No. 4 A \& B, ESA SP 1153 A \& B 1992). We selected 67 objects for which the variability properties could be investigated. For each object we estimated the amplitude of the variability by means of the standard deviation $\sigma(\lambda)$ of the flux at different frequencies divided by the mean flux.

We characterise the variability as a function of wavelength using a linear representation:

$$
\sigma(\lambda) \approx \nabla_{\lambda}\left(\sigma_{f}\right) \cdot \frac{\lambda-2000 \AA}{1000}+\sigma_{f, 2000}
$$

$\sigma_{f, 2000}$ is the variability at $2000 \AA$ expressed as a fraction of the mean flux and $\nabla_{\lambda}\left(\sigma_{f}\right)$ is the wavelength gradient of the variability in fraction $/(1000 \AA)$.

The main conclusions of this study are:

- $\sigma_{f, 2000}$ (the variability) is found to be larger than $20 \%$ in about $80 \%$ of the objects. Variability appears to be the rule in the UV domain.

- Most of the objects have a negative value of $\nabla_{\lambda}\left(\sigma_{f}\right)$, indicating that the variability is larger at small wavelength than at long wavelength.

- We tried to compare the distributions of $\sigma_{f, 2000}$ and $\nabla_{\lambda}\left(\sigma_{f}\right)$ among different subclasses of AGN: BL Lac objects, Seyfert 1 galaxies, radio-quiet and radioloud quasars. The distribution of $\sigma_{f, 2000}$ is probably different for BL Lacs objects from the one of the other subclasses, but mean variabilities are within $1 \sigma$ from each other. Mean values of $\nabla_{\lambda}\left(\sigma_{f}\right)$ are also within $1 \sigma$ from each other.

- We examinated the relation between $\sigma_{f, 2000}$ and the luminosity. We found that there is a weak trend for the variability to decrease with the increase of the luminosity. The correlation coefficient indicates that the trend has a 90 $\%$ probability of being real. In this case, the mean variability changes from $42 \%$ to $31 \%$ from low to high luminosity AGN over 5 decades. Even if this correlation is confirmed, the small value of the slope makes the models in which the luminosity is due to a number of independent events (supernovae, outbursts) inconsistent with the observations. In these models the variability should decrease with the square root of the luminosity.

A more complete study, based on ULDA 4.0, complete until the end of 1991 , is in progress and will appear in a paper. 